\title{
Development of the Automated Primer Design Workflow Uniqprimer and Diagnostic Primers for the Broad-Host-Range Plant Pathogen Dickeya dianthicola
}

\author{
Shaista Karim ${ }^{1}$, R. Ryan McNally ${ }^{1}$, Afnan S. Nasaruddin ${ }^{1}$, Alexis DeReeper ${ }^{2}$, Ramil P. Mauleon ${ }^{3}$, Amy O. Charkowski ${ }^{1}$, \\ Jan E. Leach ${ }^{1}$, Asa Ben-Hur ${ }^{4}$, and Lindsay R. Triplett ${ }^{5, \dagger}$ \\ ${ }^{1}$ Colorado State University, Department of Bioagricultural Science and Pest Management, Fort Collins, CO 80523, U.S.A. \\ ${ }^{2}$ L'Institut de recherche pour le développement, IRD, Université Montpellier, IPME, Montpellier, France \\ ${ }^{3}$ International Rice Research Institute, Los Baños, Laguna, Philippines \\ ${ }^{4}$ Colorado State University, Department of Computer Science, Fort Collins, CO 80523, U.S.A. \\ ${ }^{5}$ The Connecticut Agricultural Experiment Station, New Haven, CT 06511, U.S.A.
}

\begin{abstract}
Uniqprimer, a software pipeline developed in Python, was deployed as a user-friendly internet tool in Rice Galaxy for comparative genome analyses to design primer sets for PCRassays capable of detecting target bacterial taxa. The pipeline was trialed with Dickeya dianthicola, a destructive broad-host-range bacterial pathogen found in most potatogrowing regions. Dickeya is a highly variable genus, and some primers available to detect this genus and species exhibit common diagnostic fail-

specific and sensitive when tested with $D$. dianthicola. The remaining four amplified a minority of the nontarget strains tested. The two promising candidate primer sets were trialed with DNA isolated from 116 field samples from across the United States that were previously submitted for testing. D. dianthicola was detected in 41 samples, demonstrating the applicability of our detection primers and suggesting widespread occurrence of $D$. dianthicola in North America.
\end{abstract} ures. Upon uploading a selection of target and nontarget genomes, six primer sets were rapidly identified with Uniqprimer, of which two were
Keywords: blackleg, diagnostics, Galaxy, primer design
Bacterial plant diseases cause large global financial losses for numerous crop species. Pathogen detection is one of the most important management tools for both endemic and invasive bacterial diseases because it allows growers and regulators to exclude infected planting material and to avoid planting in contaminated soil or irrigating with contaminated water. At state and national borders, bacterial detection is crucial for identifying and quarantining seeds or other plant propagative materials infected with exotic bacterial pathogens. Cost-sensitive and time-sensitive decisions about crop destruction or quarantine may hinge on diagnostic test results; therefore, the tests used must be sensitive (i.e., able to detect all strains of the target group), specific (does not produce false-positive results for nontarget

${ }^{\dagger}$ Corresponding author: L. Triplett; lindsay.triplett@ct.gov

Shaista Karim and R. Ryan McNally contributed equally to this work.

Funding: We acknowledge the following agencies for funding support and sharing samples: CARE project 2017-68008-26731, Animal and Plant Health Inspection Service, United States Department of Agriculture Agricultural Research Service, USDA Specialty Crops Research Initiative project 201751181-26827, and the Faculty Development Program at the University of Agriculture, Faisalabad, Pakistan. The Rice Galaxy (and Uniqprimer in Galaxy) development and server are supported by the Taiwan Council of Agriculture Grant to IRRI, International Rice Informatics Consortium, IRD, the CGIAR Excellence in Breeding Platform, and the Genomic Open-source Breeding Informatics Initiative project. Any opinions, findings, conclusions, or recommendations expressed in this publication are those of the authors and do not necessarily reflect the views of the US Department of Agriculture.

*The $\boldsymbol{e}$-Xtra logo stands for "electronic extra" and indicates that one supplementary file is published online.

The author(s) declare no conflict of interest.

Accepted for publication 14 May 2019.

(C) 2019 The American Phytopathological Society groups), and rapid. Widely used molecular tools for bacterial phytopathogen detection include enzyme-linked immunosorbent assay (ELISA), PCR, and isothermal detection methods such as recombinase polymerase amplification (RPA) and loop-mediated isothermal amplification (LAMP), or a combination of these methods. Of these, PCR and other DNA-based methods are preferred for their sensitivity, specificity, and the ease with which the required reagents can be obtained. Thousands of available pathogen genomes have enabled comparative genomic analyses as a means to design PCR-based detection assays, and more than one dozen published pipelines or programs have been designed for genome-based differential primer designs. Alignment-based pipelines such as KPATH, Insignia, and TOPSI generate and parse alignments of whole genomes or coding sequences to identify signatures unique to the diagnostic targets (Phillippy et al. 2009; Slezak et al. 2003; Vijaya Satya et al. 2010). Alignment-free approaches, such as RUCS, PrimerHunter, PriMux, and the python package find_differential_primers.py, design primers or probes comprehensively or at intervals across a reference genome before screening them for target specificity across genomes (Duitama et al. 2009; Hysom et al. 2012; Pritchard et al. 2012; Thomsen et al. 2017). Although different existing programs are designed to suit a variety of different diagnostic needs, the adoption of comparative genomics methods for plant disease assay development has been limited in scope. Many published tools require comfort with a command-line environment, whereas web tools such as PrimerBLAST or MgenomeSubtractor depend on genome annotation or are limited to the analysis of a few sequences (Shao et al. 2010; Ye et al. 2012). Some previously published web tools are no longer maintained (Frech et al. 2009; Phillippy et al. 2009; van Hijum et al. 2003). Together, these factors may have limited the widespread adoption of comparative genomics methods for primer design in bacterial phytopathology. Our goal was to develop a rapid, web-based platform to generate robust diagnostic primers from bacterial sequence data with a minimum of upstream preparation.

We developed an alignment-based primer design pipeline implemented in Python, named Uniqprimer, that combines whole-genome alignment and parsing with primer design and cross-validation. Similar 
to some previous alignment-based programs (Vijaya Satya et al. 2010), Uniqprimer uses of a series of pairwise genome alignments to identify sequences specific to diagnostic targets for primer design and screening steps. However, rather than identifying a large number of small primer "signatures," Uniqprimer isolates and designs primers based on a small number of amplicon-length genomic regions that diverge between target and nontarget genomes. In-house versions of this pipeline have been used previously for the development of several validated conventional or LAMP primer sets (Ash et al. 2014; Lang et al. 2014; Langlois et al. 2017; Triplett et al. 2011, 2015). Recently, we released an online version of Uniqprimer as one of many tools on the Rice Galaxy resource (galaxy.irri.org; Juanillas et al. 2018) hosted by the International Rice Research Institute. Galaxy is a user-friendly internet platform that facilitates performing bioinformatics tasks in a reproducible way (Goecks et al. 2010), and workflows can be performed by anyone with a laptop and an internet connection. Although Uniqprimer on Rice Galaxy has been reported (Juanillas et al. 2018), its parameters and wet laboratory validation have not been previously reported.

Here, we describe the Rice Galaxy implementation of the Uniqprimer pipeline that we used to develop diagnostic tools for Dickeya dianthicola, a broad-host-range pathogen responsible for a recent outbreak of blackleg in potato in North America (Ma et al. 2018). D. dianthicola is a pectolytic Gram-negative bacterial pathogen that colonizes potato stems and tubers and causes stem blackening, plant wilt, and tuber decay in potato (Charkowski 2018). Dozens of Dickeya sequences are currently available, are numerous genome sequences from closely related species (Zhang et al. 2016). Primer development for Dickeya species, however, has been continually problematic because of its wide genomic diversity and frequent genetic exchanges. In 2013, Pritchard et al. used a comprehensive alignment-free pipeline to design $>80$ primers for Dickeya detection, of which DIA-A and DIA-C were successfully adopted for widespread diagnostic use. A primer set that was previously reliable, DIA-A, was found to yield false-negative results for a subset of recently described North American $D$. dianthicola strains that had lost the target operon for these primers (A.O. Charkowski and N.T. Perna, unpublished data). Recently

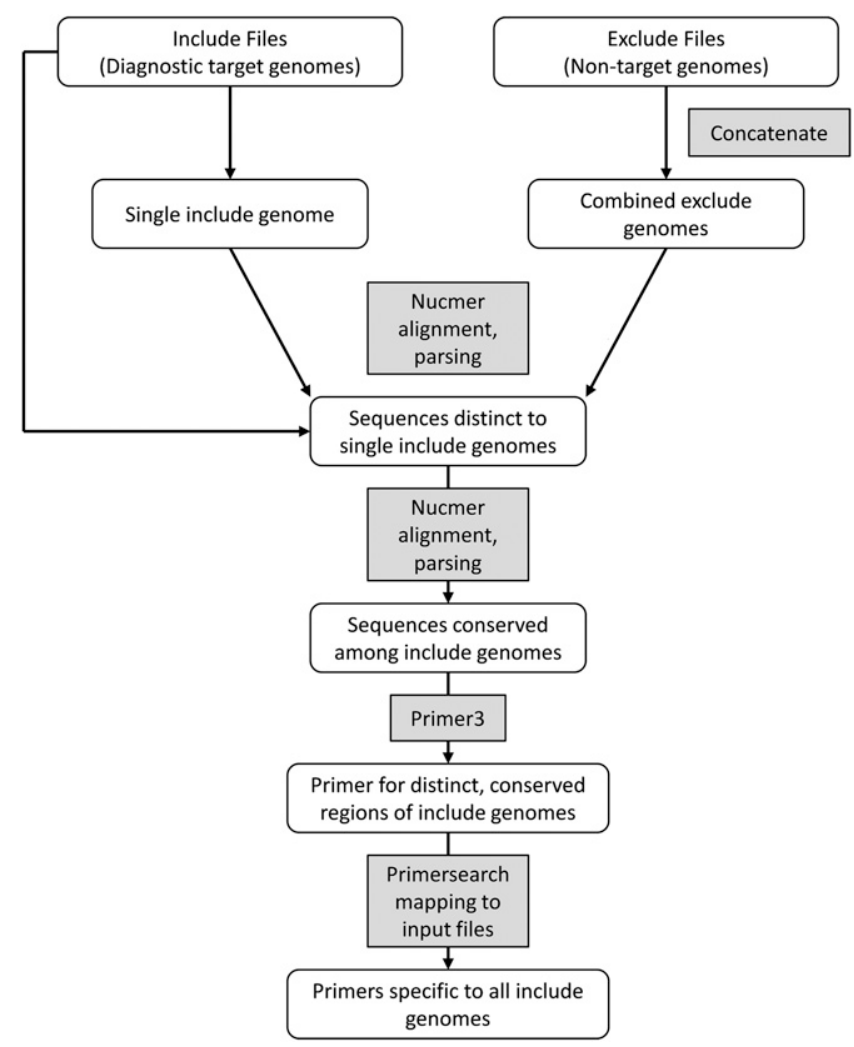

Fig. 1. Flowchart of the Uniqprimer process for primer design. White boxes represent Uniqprimer stages. Gray boxes represent analytical processes. designed primers based on the arbitrarily chosen $\operatorname{dnaX}$ failed when tested with field samples (van der Wolf et al. 2014). Therefore, the North American potato industry is currently dependent on a single primer set, DIA-C, for the diagnosis and detection of this highly variable pathogen, which is likely to result in the selection for strains that lack this target sequence. This is a common problem for diagnostic assays of variable pathogens, and it highlights the need to have multiple distinct detection tools available. To provide additional assays for the potato industry, we used Uniqprimer to develop additional primer sets that can be used with specific and sensitive assays for $D$. dianthicola detection and disease management.

\section{Materials and Methods}

Uniqprimer design and implementation on Rice Galaxy. Uniqprimer is a Python script that can be run as a stand-alone script in a Linux command line environment. Uniqprimer incorporates wholegenome alignment and parsing with primer design tools and in silico cross-validation. The Uniqprimer Python code is hosted at the Southgreen github repository (https://github.com/SouthGreenPlatform/ Uniqprimer). A functional Uniqprimer tool is publicly available via Galaxy, which is an open-source bioinformatics internet portal (Afgan et al. 2018). The graphical interface of Uniqprimer in the Rice Galaxy accepts inputs of "include" (diagnostic target) genomes and "exclude" (diagnostic nontarget) genomes, which are uploaded by the user as a single or multiFASTA sequence format files (Fig. 1). The script uses NUCmer, a suffix tree-based nucleotide alignment program in the MUMmer package, to rapidly align one of the target genomes against the combined nontarget sequences (Delcher et al. 2002). Regions of the target genome identified as mismatches for all nontarget genomes are then aligned to the additional target genomes in an iterative fashion, and regions that are not identified as matches in all target genomes are eliminated. Regions that are both distinct to and conserved among the group of target genomes are parsed into a multiFASTA sequence file. This file is used as input for primer design using the Primer3 program (Koressaar and Remm 2007). Primer3 is set at the default stringency; users can modify the primer length and product size range as desired. Finally, primers are mapped to target and nontarget genomes using the Primersearch program from the package EMBOSS (Rice et al. 2000), thereby eliminating any primers that are predicted to amplify a nontarget genome or that do not perfectly match a target genome. The output is a file of primer pair sequences including predicted product length and melting temperature $\left(\mathrm{T}_{\mathrm{m}}\right)$ and a logfile with details of the run. A step-by-step tutorial for using Uniqprimer is posted on the Rice Galaxy site (Supplemental Figure S1).

Primer design and in silico analyses. Uniqprimer was used for the design of primers specific to $D$. dianthicola. To identify relevant genomes for successful primer design, 39 complete or draft genomes representing Dickeya, Pectobacterium, and Brennaria isolates were collected from the ASAP online database (https://asap.genetics.wisc. edu) and subjected to whole-genome phylogenetic analysis using REALPHY 1.12 (Bertels et al. 2014) (Table 1 and Fig. 2). Six D. dianthicola genomes, including two draft contig assemblies, were uploaded as target genomes, and 33 other enterobacterial genomes were concatenated and uploaded as nontarget genomes (Table 1). All primer options were left on the default settings (product size range, 100 to $300 \mathrm{bp}$; optimal primer size, 20 nucleotides; minimum primer size, 18 nucleotides; and maximum primer size, 27 nucleotides). The option to cross-check primers for specificity was selected. Primer output was checked for the specificity for $D$. dianthicola among the Genbank nr and Refseq representative genomes databases using Primer-BLAST (Ye et al. 2012). D. dianthicola target genes identified through the Primer-BLAST were analyzed for conservation among Dickeya and Pectobacterium using BlastN (Altschul et al. 1990).

Source and identity of isolates and environmental samples. Bacterial strains used in this study are listed in Table 1 . The collection included multiple $D$. dianthicola strains, Dickeya species, genera related to Dickeya, and other bacteria found on potato. The bacterial strains were identified through morphology and either multi-locus 
sequence or genome sequence analyses. To confirm the specificity of our primers on diverse agricultural samples, DNA isolated from potato tissues samples and irrigation water was collected from multiple states, including California, Colorado, Florida, Michigan, Missouri, New Mexico, New York, North Carolina, Texas, and Wisconsin.

Bacterial DNA extraction. Bacterial isolates were streaked onto nutrient agar and were grown at $25^{\circ} \mathrm{C}$ for 2 days (Schaad et al. 2001). Bacterial cells were harvested from the plate, and DNA was extracted using a FastPrep kit (MP Biomedicals). To extract bacterial DNA from plant stems and tubers, a tissue sample was excised from the border of the symptomatic regions. The sample was vortexed in $400 \mu \mathrm{l}$ of sterile water, and DNA was extracted from the water with a FastDNA Spin Kit for Soil (MP Biomedicals). DNA quality and quantity were determined for DNA isolated from pure cultures and from diagnostic samples with a NanoDrop 1000 spectrophotometer (Thermo Fisher Scientific). DNA was stored at $-20^{\circ} \mathrm{C}$.

Specificity of conventional PCR and quantitative PCR assays. Purified bacterial genomic DNA was serially diluted in sterile water to concentrations ranging from $1 \mathrm{ng}$ to $100 \mathrm{fg}$, and these standards were used to determine the sensitivity of conventional PCR (cPCR) and real-time (quantitative PCR [qPCR]) assays. For cPCR, assays were considered positive if a band was visible by imaging on a $2.0 \%$ agarose gel after staining with SYBR safe DNA gel stain (Thermo Fisher Scientific); for qPCR, we used a $\mathrm{C}_{\mathrm{T}}$ value of $28.22 \pm 2$ to detect $D$. dianthicola.

For primer set DDI-1, PCR was conducted in a $25-\mu$ l reaction that contained $10 \mu \mathrm{l}$ of Q-solution (5x), $1 \mu \mathrm{l}$ of dNTP mix (10 mM), $5 \mu 1$ of reaction buffer $(10 \times), 1 \mu \mathrm{l}$ of DDI-1 primers $(10 \mu \mathrm{M}), 10 \mathrm{ng}$ of DNA template, and $0.5 \mu \mathrm{l}$ of HotstartTaq DNA polymerase ( 5 units $/ \mu \mathrm{l})$ (Table 2). Thermal cycling parameters consisted of $3 \mathrm{~min}$ at $95^{\circ} \mathrm{C}$ and $30 \mathrm{~s}$ at $95^{\circ} \mathrm{C}$, followed by 25 cycles of $30 \mathrm{~s}$ at $57^{\circ} \mathrm{C}$, extension at $72^{\circ} \mathrm{C}$ for $1 \mathrm{~min}$, and incubation for $5 \mathrm{~min}$ at $72^{\circ} \mathrm{C}$ and $10^{\circ} \mathrm{C}$ for infinite hold.

The specificity of primer set DDI-2 was evaluated using the same bacterial isolate collection that was used for cPCR testing. For qPCR, the reactions were conducted in $20 \mu \mathrm{l}$, including $1 \mu \mathrm{l}$ of primers $(10$ $\mu \mathrm{M}), 2 \mu \mathrm{l}$ of the DNA template $(50 \mathrm{ng})$, and $10 \mu \mathrm{l}$ of Power SYBR Green PCR master mix (2x) (Applied Biosystems) (Table 2). The thermal cycling conditions were as follows: $2 \mathrm{~min}$ and $30 \mathrm{~s}$ at $98^{\circ} \mathrm{C}$, followed by 30 cycles of $10 \mathrm{~s}$ for $98^{\circ} \mathrm{C}$ and $1 \mathrm{~min}$ at $60^{\circ} \mathrm{C}$ in an ABI-700 real-time PCR system (Applied Biosystems). The threshold cycles were analyzed and calculated based on absolute quantification, and values $<24 \pm 3$ were considered a positive detection response.

Table 1. Strains and genomes used in this study

\begin{tabular}{|c|c|c|c|c|c|c|c|}
\hline Species & Strain & Origin & Host & Uniqprimer $^{\mathbf{a}}$ & ASAP Genome ID & DDI-1 $^{\text {b }}$ & DDI-2 $^{b}$ \\
\hline \multicolumn{8}{|l|}{ Brenneria } \\
\hline B. goodwinii & OBR1 & $n d^{c}$ & nd & Excluded & WIS_BgoOBR1_v1.fas & - & - \\
\hline B. quercina & Equ 11D3 & California & Oak & $\mathrm{nt}^{\mathrm{d}}$ & - & - & - \\
\hline B. salicis & ATCC $15712-\mathrm{T}^{\mathrm{e}}$ & UK & Willow & Excluded & WIS_BsaATCC15712_v1.fas & - & - \\
\hline \multicolumn{7}{|l|}{ Dickeya } & - \\
\hline D. chrysanthemi & ATCC 11663-T & US & Chrysanthemum & Excluded & WIS_Dch11663_DRAFTv1.fas & - & - \\
\hline D. chrysanthemi & NCPPB 3533 & nd & nd & Excluded & WIS_Dch3533_DRAFTv1.fas & - & - \\
\hline D. chrysanthemi & M074 & Malaya & nd & Excluded & WIS_DchM074_DRAFTv1.fas & - & - \\
\hline D. dadantii & 3937 & France & African violet & Excluded & WIS_ECH3937_v6b.fas & - & - \\
\hline D. dadantii subsp. dieffenbachiae & NCPPB 2976-T & US & Dieffenbachia & Excluded & WIS_Dda2976_DRAFTv1.fas & - & - \\
\hline D. dianthicola & GBBC 2039 & Belgium & Potato & Included & WIS_Ddi2039_DRAFTv1.fas & + & + \\
\hline D. dianthicola & NCPPB 3534 & Netherlands & Potato & Included & WIS_Ddi3534_DRAFTv1.fas & + & + \\
\hline D. dianthicola & NCPPB 453-T & nd & Dianthus & Included & WIS_Ddi453_DRAFTv1.fas & + & + \\
\hline D. dianthicola & IPO 980 & Netherlands & Potato & Included & WIS_Ddi980_DRAFTv1.fas & + & + \\
\hline D. dianthicola & RNS04.9 & France & Potato & Included & WIS_DdiRNS049_DRAFTv1.fas & + & + \\
\hline D. dianthicola & CSL RW240/1 & England & nd & Included & $\begin{array}{l}\text { WIS_DicCSLRW240_ } \\
\text { DRAFTv1.fas }\end{array}$ & + & + \\
\hline D. dianthicola & IPO 1741 & Netherlands & Potato & nt & - & + & + \\
\hline D. dianthicola & IPO 3646 & Netherlands & Potato & nt & - & + & + \\
\hline D. dianthicola & IPO 502 & Netherlands & Potato & nt & - & + & + \\
\hline D. dianthicola & ME 23 & US & Potato & nt & - & + & + \\
\hline D. dianthicola & TXG 3 & US & Potato & nt & - & + & + \\
\hline D. paradisiaca & 703 & Australia & Potato & Excluded & WIS_Dic703_v3.fas & - & - \\
\hline D. paradisiaca & ATCC 33242-T & nd & Banana & Excluded & WIS_Dpa2511_DRAFTv1.fas & - & - \\
\hline D. solani & MK10 & Israel & Potato & Excluded & WIS_DsoMK10_DRAFTv1.fas & - & - \\
\hline D. solani & MK16 & UK & nd & Excluded & WIS_DsoMK16_DRAFTv1.fas & - & - \\
\hline D. solani & IPO 2222 & Netherlands & Potato & nt & - & - & - \\
\hline D. solani & IPO 2187 & Israel & Potato & $\mathrm{nt}$ & - & - & - \\
\hline D. solani & IPO 3648 & Netherlands & Potato & $\mathrm{nt}$ & - & - & - \\
\hline Dickeya sp. & NCPPB 3274 & St. Lucia & Aglaonema & Excluded & WIS_Dic3274_DRAFTv1.fas & - & - \\
\hline Dickeya sp. & NCPPB 569 & Australia & Sugarcane & Excluded & WIS_Dic569_DRAFTv1.fas & - & - \\
\hline Dickeya sp. & DW 0440 & nd & nd & Excluded & WIS_DicDW0440_DRAFTv1.fas & - & - \\
\hline Dickeya fangzhongdai & MK7 & Scotland & nd & Excluded & WIS_DicMK7_DRAFTv1.fas & - & - \\
\hline D. zeae & Ech1591 & US & Corn & nt & - & - & - \\
\hline D. zeae & Ech586 & Florida & Philodendron & Excluded & WIS_Dic586_v1.fas & - & - \\
\hline D. zeae & NCPPB 3531 & Australia & nd & Excluded & WIS_Dze3531_DRAFTv1.fas & - & - \\
\hline D. zeae & EC1 & China & Rice & Excluded & WIS_DzeEC1_v1.fas & - & - \\
\hline D. zeae & MK19 & nd & nd & Excluded & WIS_DzeMK19_DRAFTv1.fas & - & - \\
\hline
\end{tabular}

(Continued on next page)

\footnotetext{
a Status of genome in Uniqprimer analysis.

${ }^{\mathrm{b}}$ Combined results of conventional and quantitative PCR results; $+=$ positive for $D$. dianthicola detection; $-=$ no D. dianthicola detected.

${ }^{\mathrm{c}}$ Not determined.

d Not tested.

e $\mathrm{T}=$ type strain .
} 
To validate the ability of qPCR to detect $D$. dianthicola in field samples, potato tubers and stems exhibiting blackleg and soft rot symptoms were collected from farmers throughout the United States. Tissue samples were suspended in $10 \mathrm{ml}$ sterile water, and DNA was extracted using the FastDNA Spin Kit for Soil (MP Biomedicals) and stored at $-20^{\circ} \mathrm{C}$.

Sensitivity of cPCR and qPCR assays. The sensitivity of primer sets DDI-1 and DDI-2 was evaluated with $D$. dianthicola DNA. Using the template set based on pure DNA, a 10-fold series of dilutions was made by diluting a purified genomic DNA solution to concentrations from $1 \mathrm{ng}$ to $100 \mathrm{fg}$. The qPCR and cPCR were conducted with both template sets. All samples were run in three separate reactions.

\section{Results}

Description of the Uniqprimer Rice Galaxy Tool. Uniqprimer in Rice Galaxy is implemented using standard Galaxy tool development practices to wrap the Uniqprimer Python script that executes the primer design pipeline (uniqprimer.py, available at https://github. com/SouthGreenPlatform/Uniqprimer/tree/master/uniqprimer-0.5.0). The standalone python script collects the following as inputs (in the command-line parameters): FASTA files that should be included (the sequences for which you want to design a specific primer); one to several FASTA files that should be excluded (primers designed will not match these sequences); the product size range
(100 to 300 bases default), the optimal primer size (default is 20), the minimum primer size (default is 18), and the maximum primer size (default $=27$ ); and a setting to cross-validate primers by Primersearch alignment to exclude files (default is YES). The Galaxy XML tool file implements the graphical interface to capture these parameters and passes these to the Uniqprimer python script (uniqprimer.xml, available at https://github.com/SouthGreenPlatform/ Uniqprimer).

Design of $D$. dianthicola species-specific primers with Uniqprimer. Prior to the primer design, 39 genome sequences representing the Pectobacteriaceae were analyzed via whole-genome phylogenetic analysis (Fig. 2). Six genomes were confirmed or predicted to be $D$. dianthicola (Fig. 2). The six confirmed $D$. dianthicola genomes were used as inputs for the Uniqprimer analysis with 33 control genomes representing other Dickeya, Pectobacterium, and Brenneria species (Table 1 and Fig. 2). Uniqprimer analysis was executed in 9 minutes and $42 \mathrm{~s}$ in our experience; however, execution times may vary according to the number and size of input genomes, the number of initial primers screened, and the number of simultaneous users on Rice Galaxy. As part of its output, the Uniqprimer identified 24 genome regions as diagnostic candidate regions predicted to be distinct from and conserved among $D$. dianthicola. Of the initial primer pairs designed from those regions (two from each region), six primer pairs were confirmed via the Uniqprimer

Table 1. (Continued from previous page)

\begin{tabular}{|c|c|c|c|c|c|c|c|}
\hline Species & Strain & Origin & Host & Uniqprimer $^{\mathbf{a}}$ & ASAP Genome ID & DDI-1 $^{\text {b }}$ & DDI-2 ${ }^{\mathbf{b}}$ \\
\hline \multicolumn{8}{|l|}{ Pectobacterium } \\
\hline P. atrosepticum & $21 \mathrm{~A}$ & Belarus & Potato & Excluded & WIS_Pat21A_v1.fas & - & - \\
\hline P. atrosepticum & CFBP 6276 & France & Potato & Excluded & WIS_Pat6276_DRAFTv1.fas & - & - \\
\hline P. atrosepticum & SCRI1043 & UK & Potato & Excluded & WIS_SCRI1043_v2.fas & - & - \\
\hline$P$. atrosepticum & ATCC 33260 & UK & Potato & nt & - & - & - \\
\hline P. betavasculorum & ATCC 43762-T & California & Sugar beet & Excluded & WIS_Pbe43762_DRAFTv1.fas & - & - \\
\hline P. betavasculorum & Ecb 2 & nd & nd & nt & - & - & - \\
\hline P. betavasculorum & Ecb 6 & nd & nd & nt & - & - & - \\
\hline P. betavasculorum & Ecb 1 & nd & nd & nt & - & - & - \\
\hline P. carotovorum & WPP14 & Wisconsin & Potato & Excluded & WIS_EccWPP14_v2.fas & - & - \\
\hline P. carotovorum & $\mathrm{BC} 1$ & China & Chinese cabbage & Excluded & WIS_PcaBC1_v1.fas & - & - \\
\hline P. carotovorum & ATTCC 15713 & Denmark & Potato & nt & - & - & - \\
\hline P. carotovorum subsp. brasiliensis & 1692 & Brazil & Potato & Excluded & WIS_WPP1692_v2.fas & - & - \\
\hline P. carotovorum subsp. brasiliensis & WPP165 & Wisconsin & Potato & nt & - & - & - \\
\hline P. carotovorum subsp. brasiliensis & WPP5 & Wisconsin & Potato & $\mathrm{nt}$ & - & - & - \\
\hline P. carotovorum subsp. brasiliensis & WPP20 & Wisconsin & Potato & nt & - & - & - \\
\hline P. carotovorum subsp. brasiliensis & WPP1 & Wisconsin & Potato & nt & - & - & - \\
\hline $\begin{array}{l}\text { P. carotovorum subsp. } \\
\text { carotovorum }\end{array}$ & $\mathrm{PCC} 21$ & Korea & Chinese cabbage & Excluded & WIS_PcaPCC21_v1.fas & - & - \\
\hline $\begin{array}{l}P . \text { carotovorum subsp. } \\
\text { carotovorum }\end{array}$ & WPP127 & Wisconsin & Potato & nt & - & - & - \\
\hline $\begin{array}{l}P . \text { carotovorum subsp. } \\
\text { carotovorum }\end{array}$ & WPP156 & Wisconsin & Potato & nt & - & - & - \\
\hline $\begin{array}{l}P . \text { carotovorum subsp. } \\
\text { carotovorum }\end{array}$ & WPP2 & Wisconsin & Potato & nt & - & - & - \\
\hline $\begin{array}{l}P . \text { carotovorum subsp. } \\
\text { carotovorum }\end{array}$ & WPP12 & Wisconsin & Potato & nt & - & - & - \\
\hline $\begin{array}{l}P . \text { carotovorum subsp. } \\
\text { carotovorum }\end{array}$ & WPP16 & Wisconsin & Potato & nt & - & - & - \\
\hline $\begin{array}{l}P . \text { carotovorum subsp. } \\
\text { carotovorum }\end{array}$ & WPP17 & Wisconsin & Potato & nt & - & - & - \\
\hline P. carotovorum subsp. odoriferum & BC S7 & nd & nd & Excluded & WIS_PcaBCS7_DRAFTv1.fas & - & - \\
\hline P. parmentieri & RNS 08.42.1A-T & France & Potato & Excluded & WIS_PpaRNS08421A_v1.fas & - & - \\
\hline$P$. parmentieri & WPP163 & Wisconsin & Potato & Excluded & WIS_PwaWPP163_v1.fas & - & - \\
\hline Pectobacterium aroidearum & PC1 & Israel & Ornithogalum & Excluded & WIS_PcaPC1_v2.fas & - & - \\
\hline P. wasabiae & ATCC 43316-T & Japan & Wasabi & Excluded & WIS_Pwa43316_v1.fas & - & - \\
\hline P. wasabiae & WPP172 & Denmark & Potato & nt & - & - & - \\
\hline $\begin{array}{l}\text { P. wasabiae } \\
\text { Other }\end{array}$ & WPP161 & Wisconsin & Potato & $\mathrm{nt}$ & - & - & - \\
\hline E. amylovora & Ea 246 & Illinois & Raspberry & nt & - & - & - \\
\hline E. amylovora & Ea 321 & France & Hawthorne & nt & - & - & - \\
\hline E. amylovora & Ea 273 & New York & Apple & $\mathrm{nt}$ & - & - & - \\
\hline
\end{tabular}


cross-check step to match all target genomes, but no excluded genomes were provided. Uniqprimer generated an output file of six primer pairs that were designated DDI-1 through DDI-6, with DDI-1 and DDI-4 originating from the same region and differing in only one nucleotide in the forward primer (Table 2). PrimerBLAST was used to confirm that all six pairs had strong predicted templates in $D$. dianthicola genomes, but not in any other bacterial genomes found in the RefSeq and $\mathrm{nr}$ databases. Mapping primers to target regions in PrimerBLAST, followed by BLASTn searches of the target regions against Genbank, revealed that all of the target genes identified by Uniqprimer have homologs that are widely distributed among enterobacteria, with the exception of the pehW locus (Table 2). Primers DDI-1 and DDI-4 targeted the same region of heme-binding $\mathrm{ABC}$ transporter $d d p A$ (WP_024104074). Primers DDI-3 and DDI-5 targeted different regions of the $c o a A B C D$ locus involved in pantetheine $4^{\prime}$-phosphate synthesis. These results

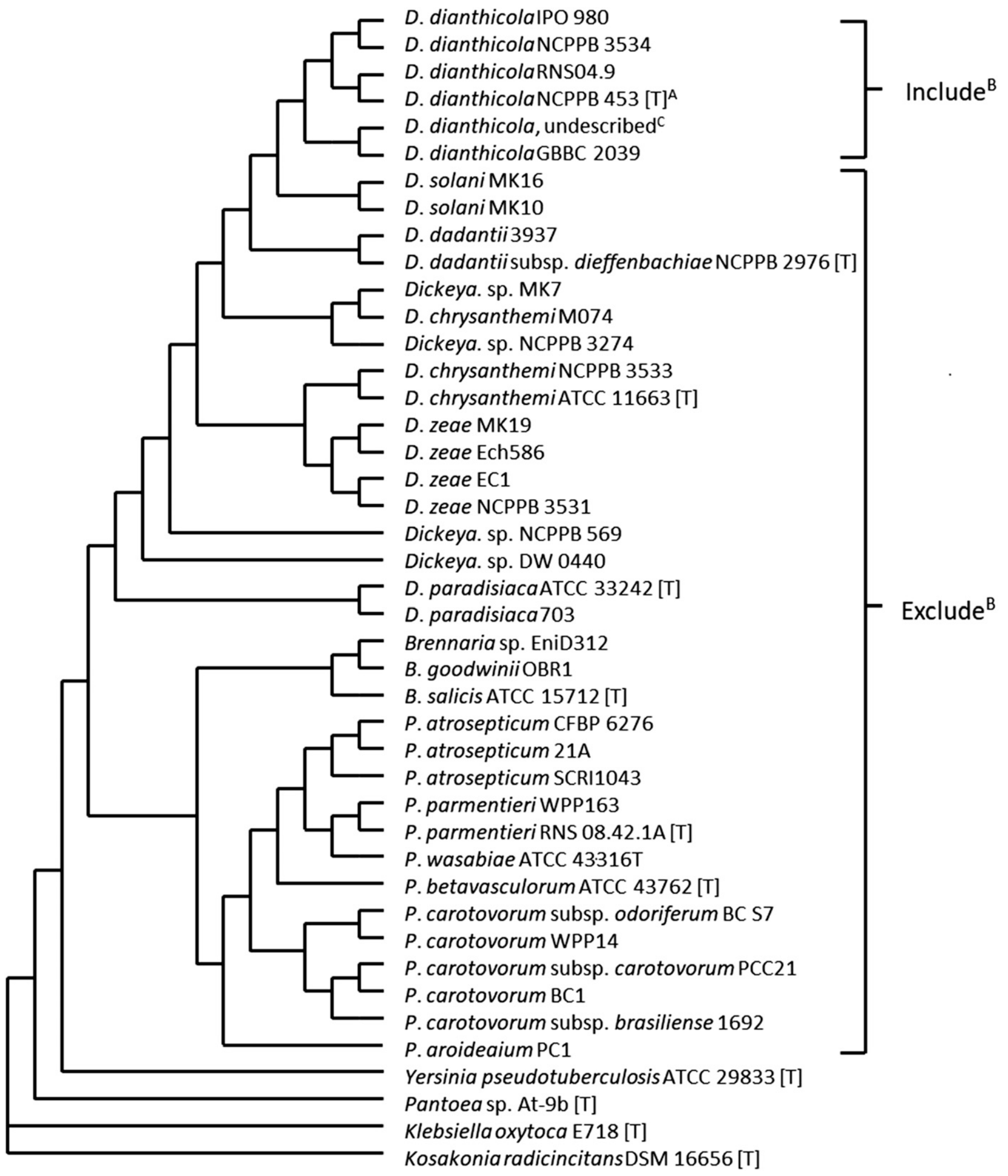

Fig. 2. Whole-genome phylogeny of Pectobacteriaceae and control strains used for Uniqprimer D. dianthicola-specific detection primer design. Tree created with REALPHY 1.12. ${ }^{A}[T]=$ type strain. ${ }^{B}$ Status in Uniqprimer analysis. CUndescribed (ASAP ID: WIS_DicCSLRW240_DRAFTv1.fas). 
Table 2. Uniqprimer output of candidate Dickeya dianthicola diagnostic primers

\begin{tabular}{|c|c|c|c|c|c|c|c|}
\hline \multirow[b]{2}{*}{ Primer } & \multirow[b]{2}{*}{ Sequence $\left(5^{\prime}-3^{\prime}\right)$} & \multirow[b]{2}{*}{ Length (bp) } & \multirow[b]{2}{*}{ Location $^{\mathbf{a}}$} & \multicolumn{3}{|c|}{ Amplified (\#/\#) } & \multirow[b]{2}{*}{ Source } \\
\hline & & & & Target & Nontarget $^{\mathbf{b}}$ & $\mathbf{T}_{\mathbf{m}}\left({ }^{\circ} \mathbf{C}\right)^{\mathbf{c}}$ & \\
\hline DDI-F1d & CTGACTATGCCTGCGTGAAA & 206 & $4601359-564$ & $5 / 5$ & $0 / 36$ & $55-65$ & This publication \\
\hline DDI-R1 & CGGAATCAGGCAGAACAGAT & & $(d p p A)$ & & & & \\
\hline DDI-F2 $2^{d}$ & GTATTCAGCTCCGCCACTTC & 201 & $4654393-593$ & $5 / 5$ & $0 / 36$ & $50-65$ & This publication \\
\hline DDI-R2 & TTTAACCTGACCAGCGGAGT & & $(u b i E)$ & & & & \\
\hline DDI-F3 & GAACTGCAACTGGCCAAAAT & 201 & $4618221-421$ & $5 / 5$ & $2 / 36$ & $\mathrm{nt}^{\mathrm{e}}$ & This publication \\
\hline DDI-R3 & AACGACAGGTCGCTTTCAGT & & $(\mathrm{coaD})$ & & & & \\
\hline DDI-F4 & TGACTATGCCTGCGTGAAAC & 205 & $4601359-564$ & $5 / 5$ & $1 / 36$ & nt & This publication \\
\hline DDI-R4 & CGGAATCAGGCAGAACAGAT & & $(d p p A)$ & & & & \\
\hline DDI-F5 & CTGCATCAGGAAATGCGATA & 194 & $4615678-871$ & $5 / 5$ & $5 / 36$ & nt & This publication \\
\hline DDI-R5 & GTGTTTCCCTGCAAGGTGTT & & $(\operatorname{coaBC})$ & & & & \\
\hline DDI-F6 & CCGCCATACCACAGGTTATC & 200 & DDI_4012 & $5 / 5$ & $3 / 36$ & $55-65$ & This publication \\
\hline DDI-R6 & CAGAGTCGCACCTTTTGACA & & $(p e h W)$ & & & & \\
\hline pelADE1 & GATCAGAAAGCCCGCAGCCAGAT & 420 & $3120359-765$ & $\mathrm{nt}$ & nt & - & Nassar et al. 1996 \\
\hline pelADE2 & CTGTGGCCGATCAGGATGGTTTTGTCGTGC & & (pel cluster) & & & & \\
\hline Df & AGAGTCAAAAGCGTCTTG & 130 & $4674870-5002$ & nt & nt & 60 & Laurila et al. 2010 \\
\hline Dr & TTTCACCCACCGTCAGTC & & (tRNA-Glu) & & & & \\
\hline DIA-A_F & GGCCGCCTGAATACTACATT & 100 & $1154606-708$ & nt & nt & 59 & Pritchard et al. 2013 \\
\hline DIA-A_R & TGGTATCTCTACGCCCATCA & & (oxidoreductase) & & & & \\
\hline DIA-C_F & CCAACGATTAGTCGGATCT & 100 & $1115639-733$ & $\mathrm{nt}$ & nt & 59 & Pritchard et al. 2013 \\
\hline DIA-C_R & TAGTTGGTGCCAGGTTGGTA & & (reductase) & & & & \\
\hline
\end{tabular}

a Location in chromosome of D. dianthicola RNS04.9 (GCA_000975305).

${ }^{\mathrm{b}}$ Nontarget amplification appeared 30 cycles after target amplification.

${ }^{\mathrm{c}} \mathrm{T}_{\mathrm{m}}=$ melting temperature range at which primers amplified target DNA.

${ }^{\mathrm{d}}$ Primers presented in this study.

$\mathrm{e} n \mathrm{nt}=$ not tested.

A

Dickeya

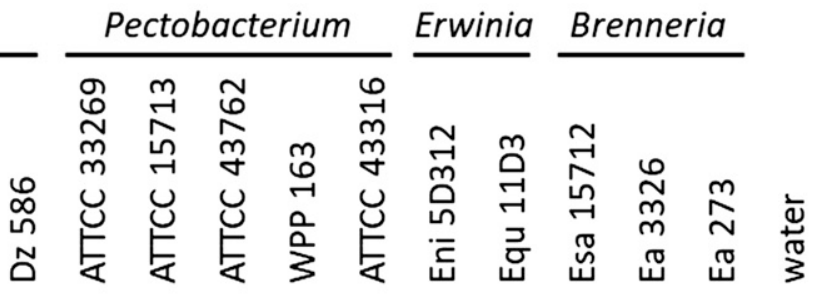

$100 \mathrm{bp}$

\begin{tabular}{|c|c|c|c|c|c|c|c|c|c|}
\hline \multicolumn{10}{|c|}{ Dickeya } \\
\hline 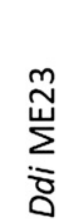 & 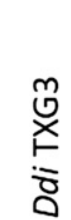 & 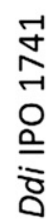 & 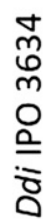 & 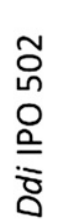 & $\begin{array}{l}\hat{n} \\
\text { ñ } \\
m \\
0 \\
\frac{0}{0}\end{array}$ & $\begin{array}{l}\text { N } \\
\text { } \\
0 \\
\frac{0}{n} \\
0\end{array}$ & 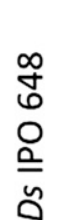 & 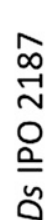 & 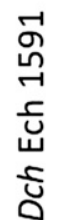 \\
\hline
\end{tabular}

B

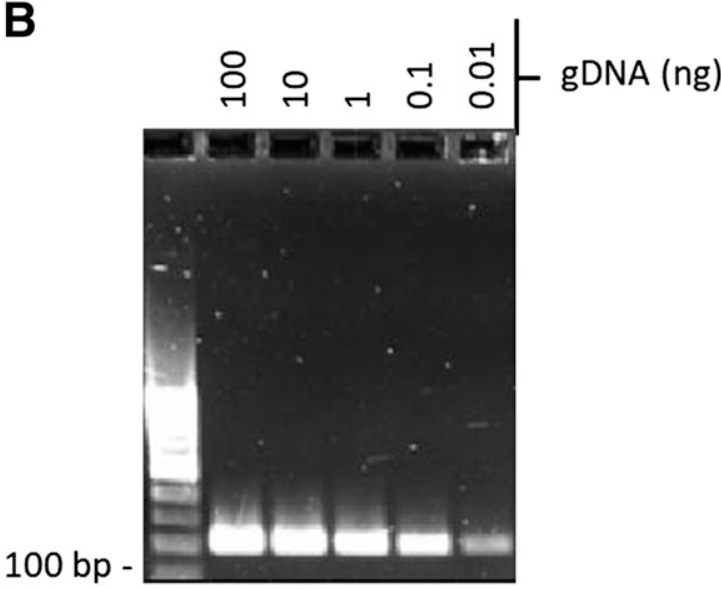

Fig. 3. Sensitivity and specificity of primers detecting $D$. dianthicola determined through conventional polymerase chain reaction (PCR). A, Amplification using DDI-1 detection primers of the targeted gene presented by 200-bp to 250-bp bands. B, Sensitivity of conventional polymerase chain reaction of pure DNA from D. dianthicola ME23. 
suggested that $d d p A$ and $c o a A B C D$ could represent regions of particular diagnostic value in Pectobacteriaceae. No regions present only in Dickeya or present only in Pectobacteriaceae were identified as primer targets by Uniqprimer.

Sensitivity and specificity of cPCR and qPCR assays. DDI primer sets identified by Uniqprimer were tested with purified DNA from $D$. dianthicola and related species. DDI-3, DDI-4, and DDI-6 primer sets amplified DNA from nontarget species in addition to $D$. dianthicola; therefore, they were excluded from subsequent analysis (Table 2). Primer sets DDI-1 and DDI-2 were sensitive to all strains of $D$. dianthicola, and no amplification was detected in other non-D. dianthicola species (Figs. 3 and 4 and Table 2). This represents a laboratory validation rate comparable with that of Pritchard et al. (2013). Using primers DDI-1 and DDI-2, neither cPCR nor qPCR amplified nontarget DNA from any of the other plant- and soilassociated bacteria that were tested, including the soft rot bacterial pathogen Pectobacterium and the closely related plant pathogenic genera Brenneria and Erwinia (Fig. 3).

Detection limit of cPCR and qPCR assays. DDI-1 and DDI-2 were tested to confirm detection limits with known $D$. dianthicola genomic DNA and field samples. To prepare for sensitivity testing, $D$. dianthicola primers were tested to confirm efficiency. Both DDI-1 and DDI-2 exhibited efficiency values within accepted limits $(100 \pm 10 \%$, Fig. 5). To determine detection limits for our assays, $100 \mathrm{ng}$ of template DNA was serially diluted to $1 \mathrm{pg}$ and tested via cPCR and $\mathrm{qPCR}$. The cPCR assays with DDI- 1 detected $D$. dianthicola DNA at $10 \mathrm{pg}$. The qPCR assays with DDI-2 exhibited detection limits down to $1 \mathrm{pg}$ of DNA template. DDI-1, DDI-2, and DIA-C consistently identified D. dianthicola in field

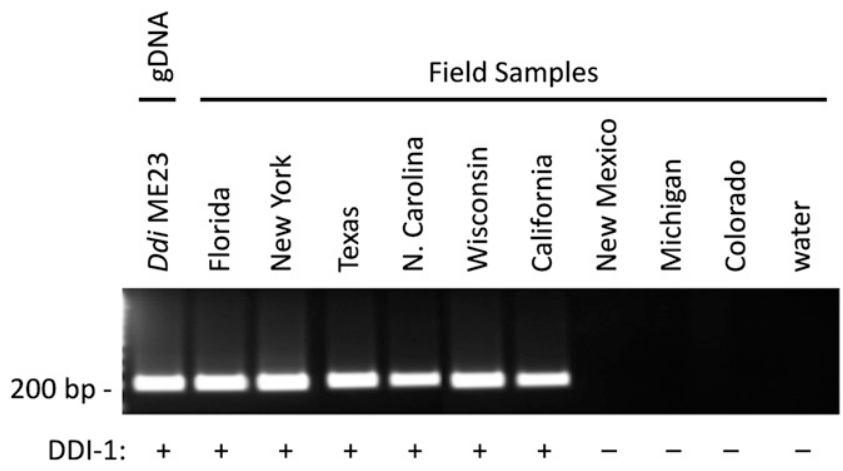

Fig. 4. Detection of Dickeya dianthicola (Ddi) in potato field samples from across the United States with genomic bacterial DNA (gDNA) control using DDI-1. $+=D$. dianthicola detected; $-=D$. dianthicola not detected.

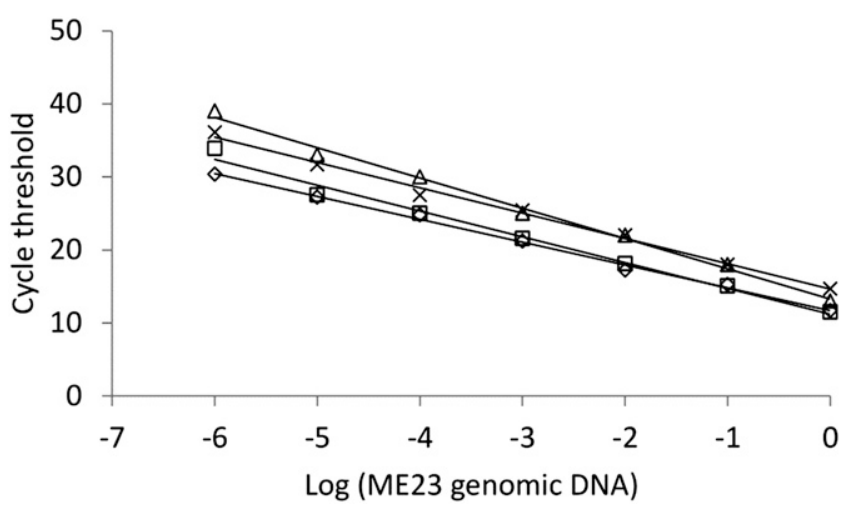

Fig. 5. Primer efficiencies calculated for quantitative polymerase chain reaction Dickeya dianthicola detection primers DDI-1 (diamond), DDI-2 (square), DIA-A (triangle), and DIA-C (cross) (Pritchard et al. 2013). Results repeated in triplicate using D. dianthicola ME23 genomic DNA template. Efficiency calculated $\left[10^{-1} /\right.$ $\mathrm{m})-1] \times 100$. samples, whereas DIA-A, pelADE, and Df/Dr inconsistently identified it in field samples, suggesting poor specificity (Table 3; Figs. 4 and 6).

Field sample analysis. To confirm the utility of our $D$. dianthicola detection primers, and to further demonstrate their specificity, we validated our Uniqprimer assays with DNA from field samples collected across the United States. In total, 52 potato stem samples, 14 potato tuber samples, 9 potato tissue culture samples, and 41 irrigation water samples from California, Colorado, Florida, Michigan, Missouri, New Mexico, New York, North Carolina, Texas, and Wisconsin were tested (Table 3). D. dianthicola was detected in 30 stem and 11 tuber samples from California, Florida, Michigan, Missouri, New Mexico, New York, North Carolina, Texas, and Wisconsin. We compared the results with those of previously published assays (Fig. 6), and our results were consistent with those of DIA-C D. dianthicola detection primers (Pritchard et al. 2013).

Comparison of DDI-1 and DDI-2 with extant tools for $D$. dianthicola detection. Currently, DNA-based D. dianthicola detection relies on the use of species-specific primers DIA-A and DIA-C combined with Dickeya general primers pelADE and Df/Dr (Nassar et al. 1996; Potrykus et al. 2014; Pritchard et al. 2013). We compared our Uniqprimer assays to current diagnostic standards, DIA-A and DIA-C, in silico using a Primer-BLAST analysis of the NCBI nonredundant whole-genome database (Fig. 7). Primer-BLAST detects potential amplification products in the NCBI genomic databases (Ye et al. 2012). As expected, DDI-1, DDI-2, DIA-A, and DIA-C shared a high level of nucleotide sequence identity with their cognate targets in all available complete $D$. dianthicola genomes (Fig. 7). DDI-1 and DDI-2 also shared a lower level of identity with amplicon-size
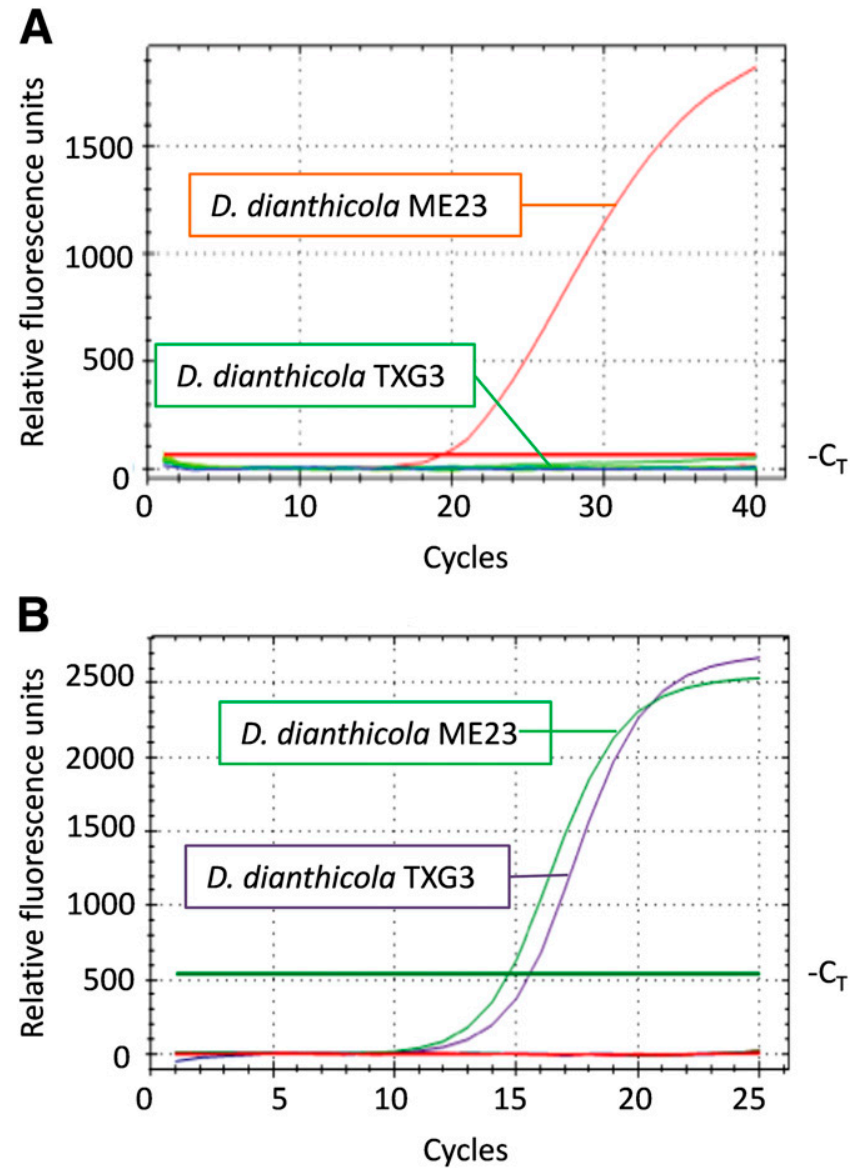

Fig. 6. Detection of Dickeya dianthicola isolates ME23 and TXG3 using a quantitative PCR (qPCR). A, qPCR detection of $D$. dianthicola isolates using diagnostic primer DIA-A (Pritchard et al. 2013). B, qPCR detection of $D$. dianthicola isolates using diagnostic primer DDI-2. $\mathrm{C}_{\mathrm{T}}=$ cycle threshold as calculated by the $\mathrm{ABI}-700$ realtime PCR system. 
regions in a greater number of off-target genomes than DIA-A or DIA-C (Fig. 7, blue shaded regions). Although this level of identity was not high enough to result in any false-positive results for DDI-1 or DD1-2 on strains tested in the laboratory (Table 1), they illustrated that the stringency settings of Uniqprimer may result in diagnostic targets that share a low level of identity with those in closely related genomes rather than targets completely missing in those genomes.

\section{Discussion}

We used the Rice Galaxy implementation of the Uniqprimer diagnostic primer design pipeline to efficiently develop primers for the sensitive and specific detection of $D$. dianthicola, a difficult-todetect pathogen that causes tuber soft rot and potato blackleg. Given a set of target and nontarget genome files, Uniqprimer generated six primer pairs with sensitivity and specificity in silico, of which two also demonstrated absolute sensitivity and specificity against a library of strains in the laboratory. Uniqprimer can be used by anyone with an internet connection and access to genome sequences of diagnostic target bacteria, although the speed of the process may vary according to the size of the dataset input and the number of simultaneous users on RiceGalaxy. Novice users can follow the supplemental tutorial to apply Uniqprimer for their own diagnostic uses. Although the in-house Python code of Uniqprimer has previously been used to develop primers for the specific detection of pathovars and geographic pathogen clades, this work demonstrated the utility of the user-friendly internet-accessible implementation of the pipeline for solving difficult diagnostic problems.

The Uniqprimer pipeline shares similarities with several previous alignment-based primer design approaches because the pipeline uses a series of rapid whole-genome alignments to isolate sequences that are distinct to and conserved among diagnostic target strains (Marinier et al. 2017; Phillippy et al. 2009; Slezak et al. 2003; Vijaya Satya et al. 2010). This is in contrast to the computational approach of alignment-free methods, which comprehensively design primer pairs at regular intervals or for all coding sequences prior to filtering. Similar to other pipelines, Uniqprimer uses the popular tools Primer3 (used in many published tools), Primersearch (used by Pritchard et al. 2012), and BLAST (Pritchard et al. 2012; Thomsen et al. 2017; Vijaya Satya et al. 2010) for primer design and screening steps. Other alignment-based programs generate a large number of primer-length signatures that are paired to primer sets after screening, but Uniqprimer generates a smaller number of amplicon-length primer design templates that share increased conservation among targets rather than between targets and nontargets. Our goal was not to compare the performance of Uniqprimer to other pipelines, but the approach of limiting the primer design to a small number of highly prefiltered regions was intended to limit the computationally intensive final screening step of aligning candidate primers to the input genomes. One benefit of isolating amplicon-length regions is that Uniqprimer output includes multiFASTA files of the candidate diagnostic target regions. This output file can be useful for nonconventional primer design, such as primers for LAMP (Ash et al. 2014).

Dickeya is a diverse genus with at least seven known species and additional subspecies that show significant genetic overlap and exchange; developing primers for species in this genus has been difficult. Various immunodetection methods and DNA-based assays have been published that describe $D$. dianthicola detection, but many lack the specificity and the sensitivity necessary to produce pathogen-free stock. We confirmed that the Df/Dr primer set resulted in false-negative results, the pelADE primer set amplified spurious DNA fragments, and the target locus of the DIA-A primer set is missing from some North American strains of $D$. dianthicola. This was unavoidable because of the unavailability of representative genomes from North America during the time of the DIA-A primer design (Nassar et al. 1996; Potrykus et al. 2014; Pritchard et al. 2013). Primers designed in this study performed comparably with the industry standard, DIA-C, with DNA from pure bacterial isolates and field samples (Pritchard et al. 2013). The remaining four resulted in false-positive results on nontarget strains in our collection, which is always a possible consequence when the sequence is unavailable for some relevant organisms. The primer pairs targeted loci that have predicted homologs in multiple genera of enterobacteria, demonstrating the utility of the pipeline for identifying distinct regions of conserved diagnostic targets that may be missed in alignment strategies targeting the presence/absence of entire loci.

To date, no primer sets are available that amplify DNA from all strains within the Dickeya genus. We also attempted to use Uniqprimer to design primers for the detection of all Dickeya species, but the pipeline failed to identify any primer pairs that did not also amplify other pectolytic or enteric bacteria. This suggested that Uniqprimer may require a certain degree of genetic closeness within the diagnostic target group, as well as a threshold of genetic distance between target and nontarget groups. Therefore, the application of Uniqprimer may be limited for groups genetically broader than the highly specific groups currently validated, including species, pathovar, and subpathovar geographic groups (Lang et al. 2014; Langlois

Table 3. Comparison of PCR detection results using Dickeya dianthicola-specific and Dickeya-general diagnostic primers

\begin{tabular}{|c|c|c|c|c|c|c|c|c|c|c|c|c|c|c|c|c|c|c|c|}
\hline \multirow[b]{2}{*}{ Sample type (n) } & \multirow[b]{2}{*}{ Location (n) } & \multicolumn{3}{|c|}{ Pel ADE } & \multicolumn{3}{|c|}{ Df/Dr } & \multicolumn{3}{|c|}{ DIA-A } & \multicolumn{3}{|c|}{ DIA-C } & \multicolumn{3}{|c|}{ DDI-1 } & \multicolumn{3}{|c|}{ DDI-2 } \\
\hline & & $+^{a}$ & $-b$ & $* \mathrm{c}$ & + & - & $*$ & + & - & $*$ & + & - & $*$ & + & - & * & + & - & * \\
\hline \multirow[t]{9}{*}{ Stems (52) } & California (2) & 0 & 2 & 0 & 0 & 2 & 0 & 0 & 2 & 0 & 0 & 2 & 0 & 0 & 2 & 0 & 0 & 2 & 0 \\
\hline & Colorado (17) & 0 & 17 & 2 & $\mathrm{nt}^{\mathrm{d}}$ & $\mathrm{nt}$ & $\mathrm{nt}$ & 0 & 17 & 0 & 0 & 17 & 0 & 0 & 17 & 0 & 0 & 17 & 0 \\
\hline & Florida (6) & 2 & 0 & 4 & 2 & 0 & 4 & 6 & 0 & 0 & 6 & 0 & 0 & 6 & 0 & 0 & 6 & 0 & 0 \\
\hline & Missouri (4) & 3 & 0 & 1 & 4 & 0 & 1 & 4 & 0 & 0 & 4 & 0 & 0 & 4 & 0 & 0 & 4 & 0 & 0 \\
\hline & New Mexico (1) & 1 & 0 & 0 & 0 & 0 & 2 & 0 & 1 & 0 & 1 & 0 & 0 & 1 & 0 & 0 & 1 & 0 & 0 \\
\hline & New York (5) & 0 & 1 & 4 & 1 & 0 & 3 & 4 & 1 & 0 & 4 & 1 & 0 & 4 & 1 & 0 & 4 & 1 & 0 \\
\hline & North Carolina (3) & 3 & 0 & 0 & 3 & $\mathrm{nt}$ & $\mathrm{nt}$ & 3 & 0 & 0 & 3 & 0 & 0 & 3 & 0 & 0 & 3 & 0 & 0 \\
\hline & Texas (2) & 2 & 0 & 0 & 2 & 0 & 0 & 0 & 2 & 0 & 2 & 0 & 0 & 2 & 0 & 0 & 2 & 0 & 0 \\
\hline & Wisconsin (12) & 3 & 5 & 4 & $\mathrm{nt}$ & $\mathrm{nt}$ & nt & 2 & 10 & 0 & 8 & 4 & 0 & 8 & 4 & 0 & 8 & 4 & 0 \\
\hline \multirow[t]{3}{*}{ Tubers (14) } & Florida (6) & 1 & 0 & 5 & 3 & 2 & 1 & 6 & 0 & 0 & 6 & 0 & 0 & 6 & 0 & 0 & 6 & 0 & 0 \\
\hline & Texas (2) & 0 & 0 & 2 & 2 & 0 & 0 & 0 & 2 & 0 & 2 & 0 & 0 & 2 & 0 & 0 & 2 & 0 & 0 \\
\hline & Wisconsin (6) & 2 & 2 & 6 & nt & $\mathrm{nt}$ & nt & 2 & 4 & 0 & 3 & 3 & 0 & 3 & 3 & 0 & 3 & 3 & 0 \\
\hline Tissue culture (9) & Colorado (9) & 0 & 9 & 0 & 0 & 9 & 0 & 0 & 9 & 0 & 0 & 9 & 0 & 0 & 9 & 0 & 0 & 9 & 0 \\
\hline \multirow[t]{3}{*}{ Water (41) } & Colorado (23) & 0 & 23 & 0 & 0 & 20 & 3 & 0 & 23 & 0 & 0 & 23 & 0 & 0 & 23 & 0 & 0 & 23 & 0 \\
\hline & Michigan (10) & 0 & 10 & 0 & 0 & 10 & 0 & 0 & 10 & 0 & 0 & 10 & 0 & 0 & 10 & 0 & 0 & 10 & 0 \\
\hline & Wisconsin (8) & 0 & 8 & 0 & $\mathrm{nt}$ & nt & $\mathrm{nt}$ & 0 & 8 & 0 & 0 & 8 & 0 & 0 & 8 & 0 & 0 & 8 & 0 \\
\hline
\end{tabular}

\footnotetext{
$\mathrm{a}+=$ positive results.

$\mathrm{b}_{-}=$negative results.

$\mathrm{c} *=$ Ambiguous results displaying faint or nonspecific banding.

${ }^{\mathrm{d}} \mathrm{nt}=$ not tested.
} 


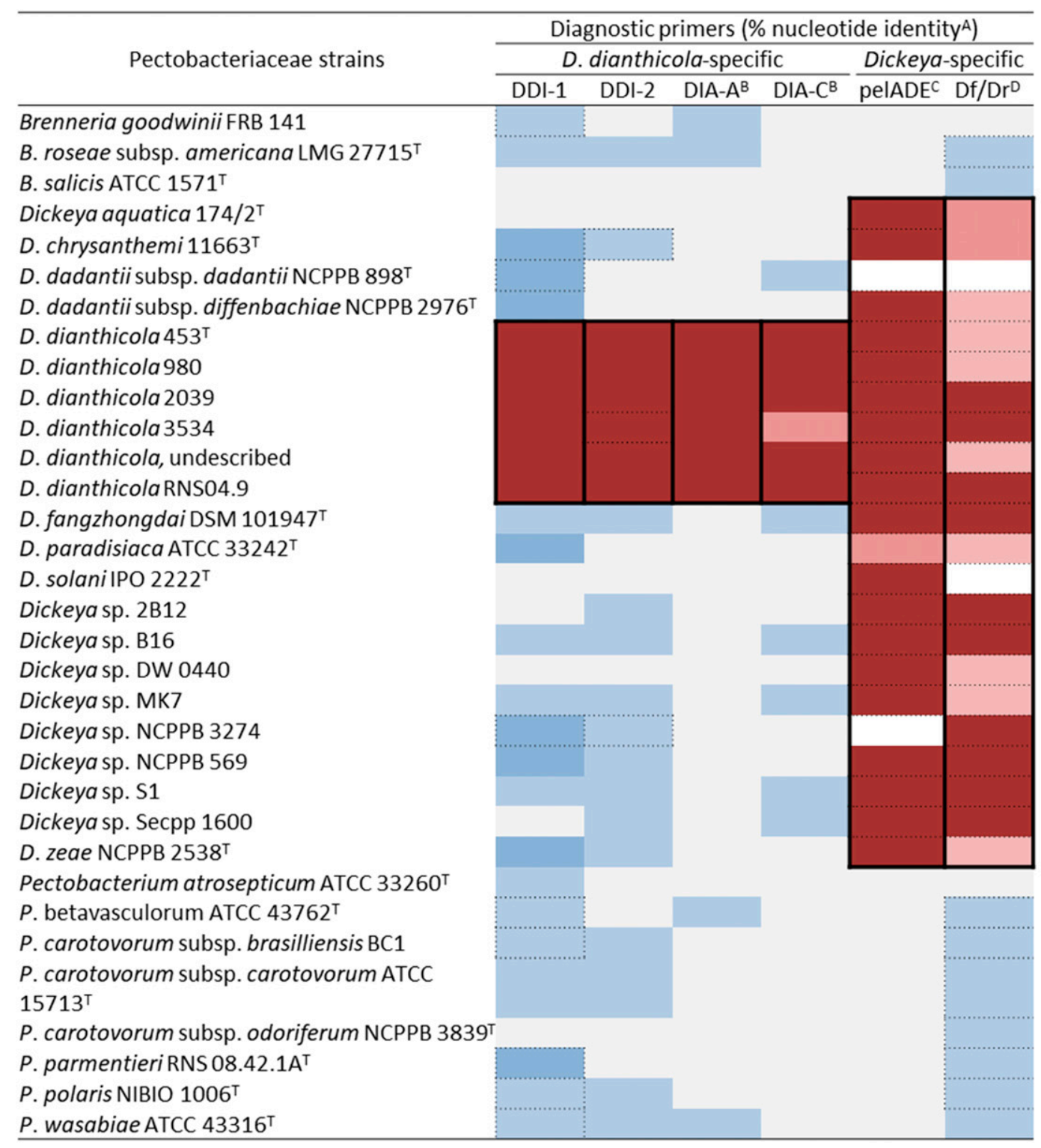

Nucleotide identity (\%)

\begin{tabular}{l|cl|l}
\hline True & $71-80$ & \\
True & $81-90$ & \\
True & $91-100$ & \\
\hline False & $91-100$ & \\
False & $81-90$ & \\
False & $71-80$ & \\
\hline
\end{tabular}

D. chrysant

D. dadantii subsp. dadantii NCPPB $898^{\top}$

D. dadantii subsp. diffenbachiae NCPPB 2976

D. dianthicola $453^{\top}$

D. dianthicola 980

D. dianthicola 2039

D. dianthicola 3534

D. dianthicola, undescribed

D. dianthicola RNS04.9

D. fangzhongdai DSM 101947

Dickeya sp. B16

Dickeya sp. DW 0440

Dickeya sp. MK

Dickeyasp. NCPPB 3274

Dickeya sp. NCPPB 569

Dickeya sp. S1

Dickeya sp. Secpp 1600

D. zeae NCPPB 2538

Pectobacterium atrosepticum ATCC $33260^{\top}$

P. betavasculorum ATCC 43762

P. carotovorum subsp. brasilliensis BC1

P. carotovorum subsp. carotovorum ATCC

P. parmentieri RNS 08.42.1 $\mathrm{A}^{\top}$

P. wasabiae ATCC $43316^{\top}$

Fig. 7. In silico analysis of Pectobacteriaceae diagnostic primers and predicted targets. A, Nucleotide conservation was determined via a Primer3-BLAST analysis of the NCBI nonredundant complete genome database. Percent nucleotide identity is presented for true-positive results (red, black border) and false-positive results (blue, gray). Results were compared with primers from B, Pritchard et al. (2013), C, Nassar et al. (1996), and D, Laurila et al. (2010). Primer-genome interactions predicted to produce multiple amplicons are noted (dotted border). ${ }^{\top}=$ Type strain.

et al. 2017). Uniqprimer prioritizes rapid and user-friendly use over exhaustive primer design; however, previously published strategies using more comprehensive alignment-free or unique signature approaches may be better suited to develop diagnostics for genuslevel detection or high-resolution differentiation of closely related strains, or to address other difficult diagnostic situations. The use of this pipeline to develop primers both distinct and comparable in performance to the $D$. dianthicola primers currently used in many testing programs that were previously generated through an alignment-free pipeline (Pritchard et al. 2013) illustrated the value of utilizing multiple complementary primer design strategies to maximize diagnostic resources. Sampling for Dickeya species has been limited to only a few crops and a few regions in the world; therefore, the discovery of new diversity and the ensuing search for new diagnostics tests will undoubtedly continue well into the future.

\section{Acknowledgments}

We thank John Herndon for the initial development work on the Uniqprimer pipeline, with funding from the Colorado State University Infectious Diseases Supercluster.

\section{Literature Cited}

Afgan, E., Baker, D., Batut, B., van den Beek, M., Bouvier, D., Čech, M., Chilton, J., Clements, D., Coraor, N., Grüning, B., Guerler, A., Hillman-Jackson, J., Jalili, V., Rasche, H., Soranzo, N., Goecks, J., Taylor, J., Nekrutenko, A., and Blankenberg, D. 2018. The Galaxy platform for accessible, reproducible and collaborative biomedical analyses: 2018 update. Nucleic Acids Res. 46 (W1):W537-W544.

Altschul, S. F., Gish, W., Miller, W., Myers, E. W., and Lipman, D. J. 1990. Basic local alignment search tool. J. Mol. Biol. 215:403-410

Ash, G. J., Lang, J. M., Triplett, L. R., Stodart, B. J., Verdier, V., Cruz, C. V., Rott, P., and Leach, J. E. 2014. Development of a genomics-based LAMP (LoopMediated Isothermal Amplification) assay for detection of Pseudomonas fuscovaginae from rice. Plant Dis. 98:909-915.

Bertels, F., Silander, O. K., Pachkov, M., Rainey, P. B., and van Nimwegen, E. 2014. Automated reconstruction of whole genome phylogenies from short sequence reads. Mol. Biol. Evol. 31:1077-1088.

Charkowski, A. O. 2018. The changing face of bacterial soft-rot diseases. Annu. Rev. Phytopathol. 56:269-288.

Delcher, A. L., Phillippy, A., Carlton, J., and Salzberg, S. L. 2002. Fast algorithms for large-scale genome alignment and comparison. Nucleic Acids Res. 30: $2478-2483$

Duitama, J., Kumar, D. M., Hemphill, E., Khan, M , Măndoiu, I. I., and Nelson, C. E.. 2009. PrimerHunter: a primer design tool for PCR-based virus subtype identification. Nucleic Acids Res. 37:2483-2492. 
Frech, C., Breuer, K., Ronacher, B., Kern, T., Sohn, C., and Gebauer, G. 2009. Hybseek: Pathogen primer design tool for diagnostic multi-analyte assays. Comput. Methods Programs Biomed. 94:152-160.

Goecks, J., Nekrutenko, A., Taylor, J., and Team, T. G. 2010. Galaxy: a comprehensive approach for supporting accessible, reproducible, and transparent computational research in the life sciences. Genome Biol. 11:R86.

Hysom, D. A., Naraghi-Arani, P., Elsheikh, M., Carrillo, A. C., Williams, P. L., and Gardner, S. N. 2012. Skip the alignment: degenerate, multiplex primer and probe design using K-mer matching instead of alignments. PLoS One 7: e34560.

Juanillas, V. M. J., Dereeper, A., Beaume, N., Droc, G., Dizon, J., Mendoza, J. R., Perdon, J. P., Mansueto, L., Triplett, L., Lang, J., Zhou, G., Ratharanjan, K., Plale, B., Haga, J., Leach, J. E., Ruiz, M., Thomson, M., Alexandrov, N., Larmande, P., Kretzschmar, T., and Mauleon, R. P. 2019. Rice Galaxy: an open resource for plant science. GigaScience 8:giz028.

Koressaar, T., and Remm, M. 2007. Enhancements and modifications of primer design program Primer3. Bioinformatics 23:1289-1291.

Lang, J. M., Langlois, P., Nguyen, M. H. R., Purdie, L., Holton, T., Djikeng, A., Cruz, C. M. V., Verdier, V., and Leach, J. E. 2014. Sensitive detection of Xanthomonas oryzae pv. oryzae and X. oryzae pv. oryzicola by Loop Mediated Isothermal Amplification. Appl. Environ. Microbiol. 80:4519-4530.

Langlois, P. A., Snelling, J., Hamilton, J. P., Bragard, C., Koebnik, R., Verdier, V., Triplett, L. R., Blom, J., Tisserat, N. A., and Leach, J. E. 2017. Characterization of the Xanthomonas translucens complex using draft genomes, comparative genomics, phylogenetic analysis, and diagnostic LAMP assays. Phytopathology 107:519-527.

Laurila, J., Hannukkala, A., Nykyri, J., Pasanen, M., Hélias, V., Garlant, L., and Pirhonen, M. 2010. Symptoms and yield reduction caused by Dickeya spp. strains isolated from potato and river water in Finland. Eur. J. Plant Pathol. 126:249-262.

Ma, X., Schloop, A., Swingle, B., and Perry, K. L. 2018. Pectobacterium and Dickeya responsible for potato blackleg disease in New York State in 2016. Plant Dis. 102:1834-1840.

Marinier, E., Zaheer, R., Berry, C., Weedmark, K. A., Domaratzki, M., Mabon, P., Knox, N. C., Reimer, A. R., Graham, M. R., Chui, L., Patterson-Fortin, L., Zhang, J., Pagotto, F., Farber, F., Mahony, J., Seyer, K., Bekal, S., Tremblay, C., Isaac-Renton, J., Prystajecky, N., Chen, J., Slade, P., and Van Domselaar, G. 2017. Neptune: a bioinformatics tool for rapid discovery of genomic variation in bacterial populations. Nucleic Acids Res. 45:e159.

Nassar, A., Darrasse, A., Lemattre, M., Kotoujansky, A., Dervin, C., Vedel, R., and Bertheau, Y. 1996. Characterization of Erwinia chrysanthemi by pectinolytic isozyme polymorphism and restriction fragment length polymorphism analysis of PCR-amplified fragments of pel genes. Appl. Environ. Microbiol. 62:2228-2235.

Phillippy, A. M., Ayanbule, K., Edwards, N. J., and Salzberg, S. L. 2009. Insignia: a DNA signature search web server for diagnostic assay development. Nucleic Acids Res. 37 (Web Server):W229-W234.

Potrykus, M., Sledz, W., Golanowska, M., Slawiak, M., Binek, A., Motyka, A., Zoledowska, S., Czajkowski, R., and Lojkowska, E. 2014. Simultaneous detection of major blackleg and soft rot bacterial pathogens in potato by multiplex polymerase chain reaction. Ann. Appl. Biol. 165:474-487.

Pritchard, L., Holden, N. J., Bielaszewska, M., Karch, H., and Toth, I. K. 2012. Alignment-free design of highly discriminatory diagnostic primer sets for Escherichia coli O104:H4 outbreak strains. PLoS One 7:e34498.

Pritchard, L., Humphris, S., Saddler, G., Parkinson, N. M., Bertrand, V., Elphinstone, J. G., and Toth, I. K. 2013. Detection of phytopathogens of the genus Dickeya using a PCR primer prediction pipeline for draft bacterial genome sequences. Plant Pathol. 62:587-596.

Rice, P., Longden, I., and Bleasby, A. 2000. EMBOSS: the European molecular biology open software suite. Trends Genet. 16:276-277.

Vijaya Satya, R. V., Kumar, K., Zavaljevski, N., and Reifman, J. 2010. A highthroughput pipeline for the design of real-time PCR signatures. BMC Bioinformatics 11:340.

Schaad, N. W., Jones, J. B., and Chun, W. 2001. Laboratory Guide for Identification of Plant Pathogenic Bacteria, 3rd Ed. American Phytopathological Society Press, St. Paul, MN, USA.

Shao, Y., He, X., Harrison, E. M., Tai, C., Ou, H.-Y., Rajakumar, K., and Deng, Z. 2010. mGenomeSubtractor: a web-based tool for parallel in silico subtractive hybridization analysis of multiple bacterial genomes. Nucleic Acids Res. 38 (suppl_2):W194-W200.

Slezak, T., Kuczmarski, T., Ott, L., Torres, C., Medeiros, D., Smith, J., Truitt, B., Mulakken, N., Lam, M., Vitalis, E., Zemla, A., Ecale Zhou, C., and Gardner, S. 2003. Comparative genomics tools applied to bioterrorism defence. Brief. Bioinform. 4:133-149.

Thomsen, M. C. F., Hasman, H., Westh, H., Kaya, H., and Lund, O. 2017. RUCS rapid identification of $\mathrm{PCR}$ primers for unique core sequences. Bioinformatics 33:3917-3921.

Triplett, L. R., Hamilton, J. P., Buell, C. R., Tisserat, N. A., Verdier, V., Zink, F., and Leach, J. E. 2011. Genomic analysis of Xanthomonas oryzae isolates from rice grown in the United States reveals substantial divergence from known X. oryzae pathovars. Appl. Environ. Microbiol. 77:3930-3937.

Triplett, L. R., Verdier, V., Campillo, T., Van Malderghem, C., Cleenwerck, I., Maes, M., Deblais, L., Corral, R., Koita, O., Cottyn, B., and Leach, J. E. 2015. Characterization of a novel clade of Xanthomonas isolated from rice leaves in Mali and proposal of Xanthomonas maliensis sp. nov. Antonie van Leeuwenhoek 107:869-881.

van der Wolf, J. M., de Haas, B. H., van Hoof, R., de Haan, E. G., and van den Bovenkamp, G. W. 2014. Development and evaluation of Taqman assays for the differentiation of Dickeya (sub)species. Eur. J. Plant Pathol. 138:695-709.

van Hijum, S. A. F. T., de Jong, A., Buist, G., Kok, J., and Kuipers, O. P. 2003. UniFrag and GenomePrimer: selection of primers for genome-wide production of unique amplicons. Bioinformatics 19:1580-1582.

Ye, J., Coulouris, G., Zaretskaya, I., Cutcutache, I., Rozen, S., and Madden, T. L. 2012. Primer-BLAST: A tool to design target-specific primers for polymerase chain reaction. BMC Genomics 13:134-144.

Zhang, Y., Fan, Q., and Loria, R. 2016. A re-evaluation of the taxonomy of phytopathogenic genera Dickeya and Pectobacterium using whole-genome sequencing data. Syst. Appl. Microbiol. 39:252-259. 